\title{
Variations in soil microbial community diversity and structure under different types of degradation wetland in Sanjing Plain, northeastern China
}

\author{
Yining $\mathrm{Wu}^{1}$, Nan $\mathrm{Xu}^{2}$, He Wang ${ }^{1}$, Jinbo $\mathrm{Li}^{3}$, Haixiu Zhong ${ }^{3}$, and Cheng Zong ${ }^{1}$ \\ ${ }^{1}$ Northeast Forestry University \\ ${ }^{2}$ Haerbin University \\ ${ }^{3}$ Heilongjiang Academy of Sciences Institute of Natural Resources and Ecology
}

September 11, 2020

\begin{abstract}
Sanjiang Plain is the largest area of freshwater wetland in China. Due to agricultural development, a large volume of groundwater in this area has been extracted over the last few decades, resulting in degradation of the wetland. In order to provide information for the development and protection of wetland ecosystems, investigations examining processes of wetland degradation are important. The aim of this work was to assess the effects of wetland degradation on soil microbial communities and soil characteristics under four different types of degradation wetland including swamp meadow (SW), meadow wetland (MW), paddy farmland (PF) and cropland (CL) in Sanjiang Plain. Using both 16S and ITS rRNA gene amplicon sequencing to analyze soil bacterial and fungal diversity and composition. The predominant bacterial and fungal phyla were Proteobacteria and Ascomycota in this study, respectively. Additionally, wetland degradation significantly increased the relative abundances of Chloroflexi and Gemmatimonadetes, but the relative abundances of Proteobacteria and Verrucomicrobia significantly decreased. Bacterial Shannon index of SW were lower than those in other sites. While, fungal diversity had no significant differences under different types of degradation wetland. Along with the wetland degradation, such differential responses of the microbial diversity and dominant phyla were significantly correlated with TP, TK, AK and SOM, which were the most important factors affecting soil microbial communities. Generally, these results suggested that wetland degradation could result in variations in soil microbial community composition structure. These changes could be used as an early warning signal for the degradation wetland in Sanjiang Plain.
\end{abstract}

Variations in soil microbial community diversity and structureunderdifferent types ofdegradation wetland in Sanjiang Plain, northeastern China

Yining $\mathrm{Wu}^{1,2}$, Nan $\mathrm{Xu}^{1,3}$, He Wang ${ }^{1}$, Jinbo $\mathrm{Li}^{2}$, Haixiu Zhong ${ }^{2}$, Cheng Zong ${ }^{1}$

Northeast Forestry University, Heilongjiang Academy of Sciences Institute of Natural Resources and Ecology, Harbin University

Correspondence

Cheng Zhong, Northeast Forestry University, Hexing Road, 26, Harbin, Heilongjiang 150040, PR China.

Email: moredonkey@163.com

\section{Hosted file}

degradation_wetland_0904-1.docx available at https://authorea.com/users/357426/articles/ 479971-variations-in-soil-microbial-community-diversity-and-structure-under-different- 
types-of-degradation-wetland-in-sanjing-plain-northeastern-china
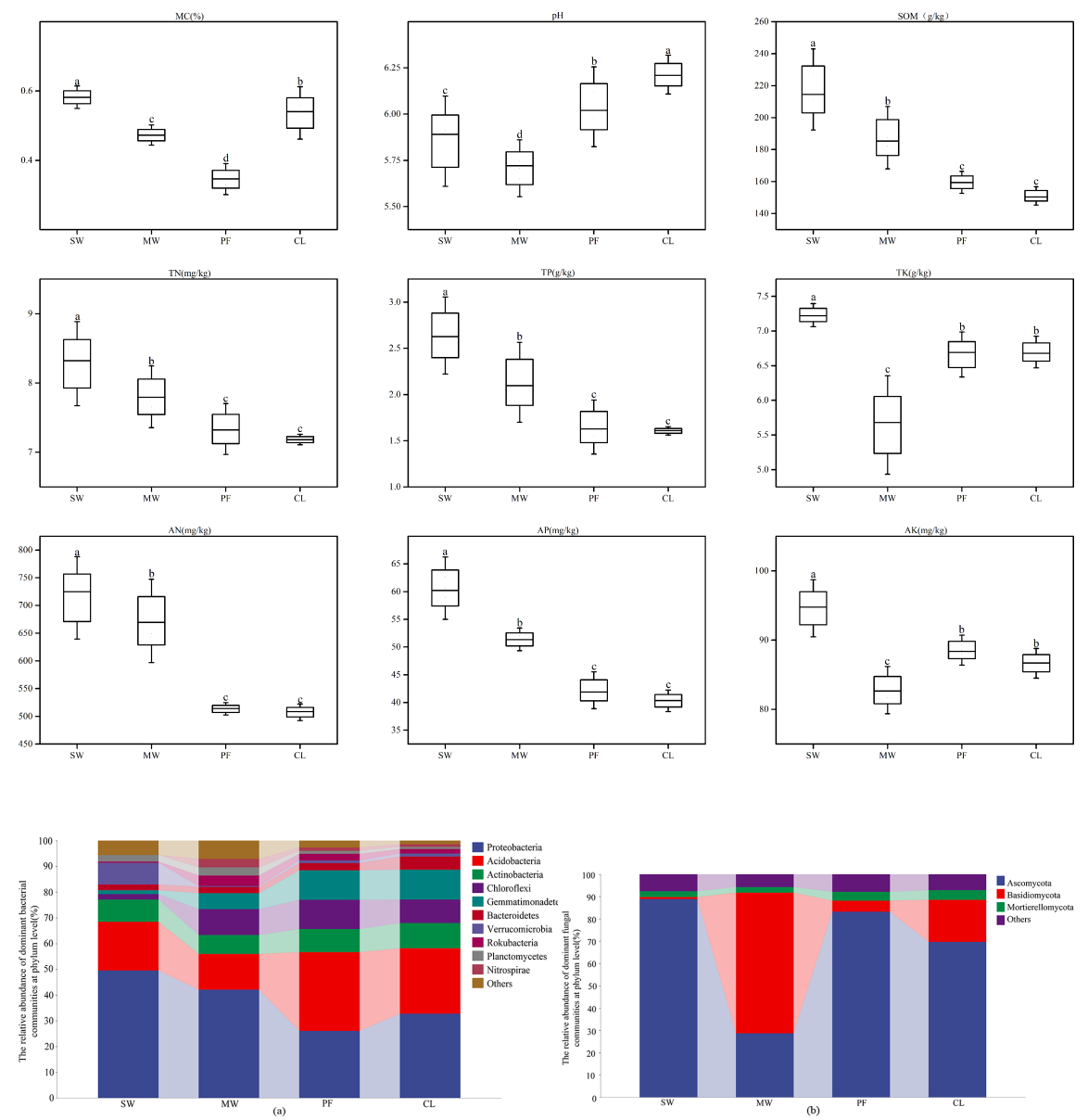

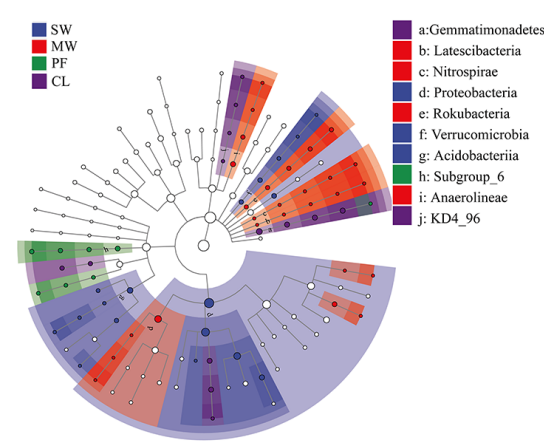

(a)

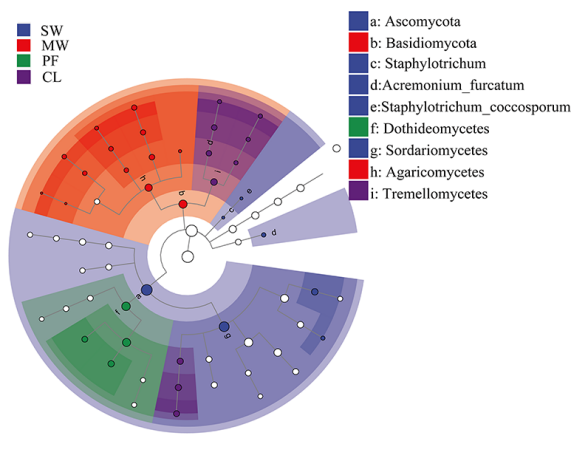

(b) 

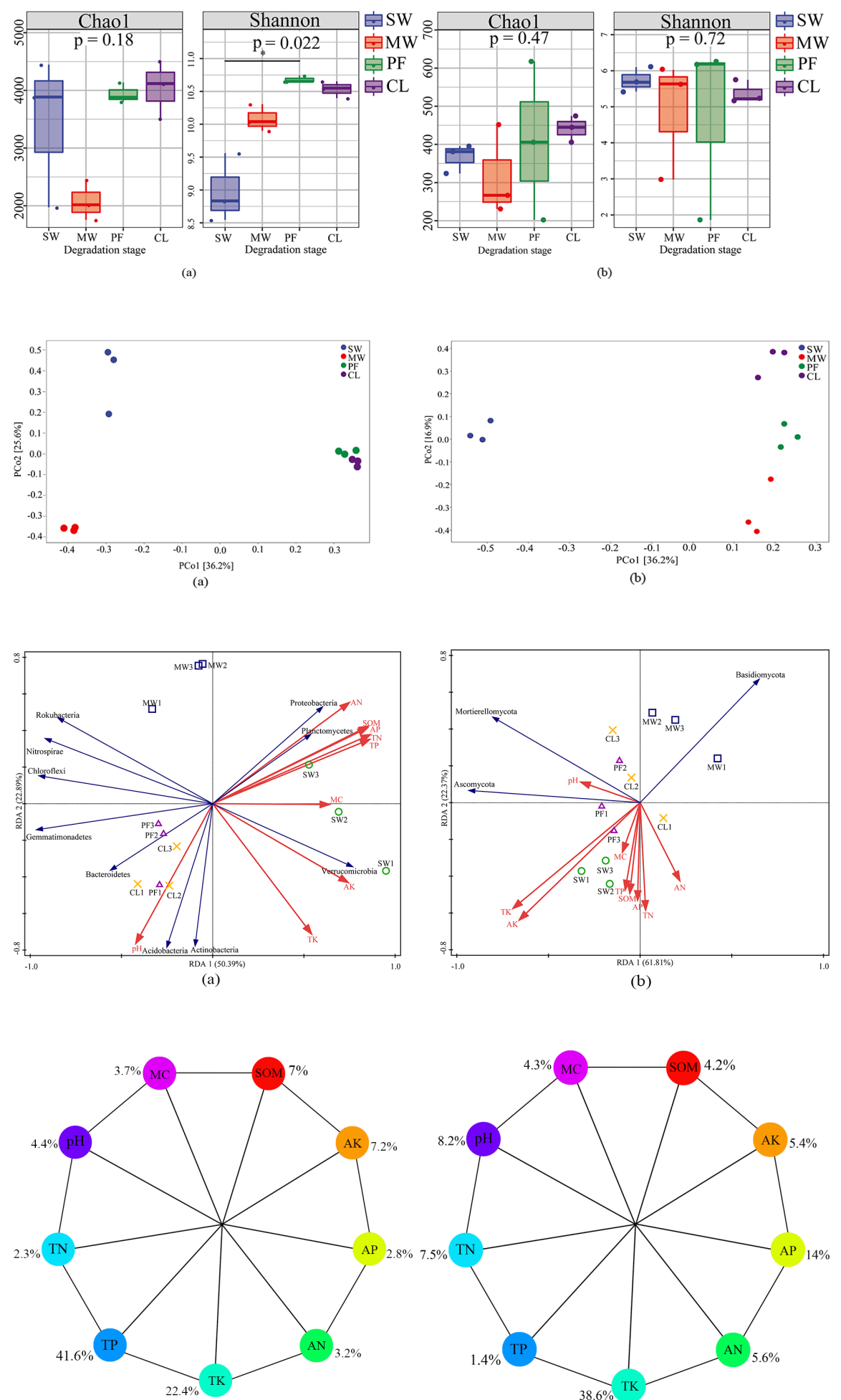

(a)

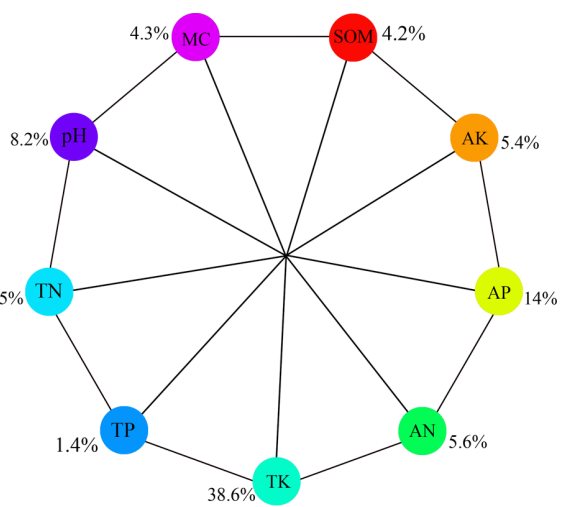

(b) 